\title{
Consumo de alcohol y drogas y factores psicosociales asociados en adolescentes de Lima
}

\author{
EDUARDO SALAZAR ${ }^{1}$, MANUEL UGARTE ${ }^{1}$, LUIS VÁSQUEZ2 ${ }^{2}$, JOSÉ LOAIZA ${ }^{2}$ \\ ${ }^{1}$ Médico SERUMS, Fuerza Aérea del Perú. Lima, Perú. ${ }^{2}$ Médico SERUMS, Ejército Peruano. Lima, Perú.
}

\begin{abstract}
Resumen
Objetivo: Determinar la prevalencia del consumo de alcohol y drogas y los factores psicosociales asociados con dicho consumo en adolescentes de Lima. Diseño: Estudio analítico de corte transversal. Material y Métodos: Se estudió una muestra de 430 adolescentes de educación secundaria de un colegio de Lima. Se utilizó el Cuestionario Tamiz de Experiencias Personales (Personal Experience Screening Questionnaire - PESQ). Resultados: Se recibió 391 encuestas válidas. La prevalencia de consumo de alcohol o drogas fue 43\%, de alcohol 42,2\%, de marihuana $8,7 \%$ y de cocaína $3,1 \%$. Se encontró problema de consumo de alcohol o drogas en el 10,7\%, los factores psicosociales asociados fueron distrés psicológico severo (OR 4,58 IC 95\% 1,60-13,05), pensamiento problemático severo (OR 2,63 IC 95\% 1,15-6,01) y abuso físico (OR 2,31 IC 95\% 1,19-4,49). Conclusiones: El factor psicosocial más asociado al problema de consumo de drogas fue el distrés psicológico severo. Asimismo, se encontró asociación entre el pensamiento problemático severo y el abuso físico con dicho problema. El acoso sexual sólo se encontró asociado en los adolescentes de segundo de secundaria.
\end{abstract}

Palabras clave: Dependencia a sustancias; drogas ilícitas; abuso de sustancias; problemas sociales; alcoholismo.

\begin{abstract}
Alcohol and drugs consumption and associated psychosocial factors in Lima adolescents

Objective: To determine the prevalence of alcohol and drug use and psychosocial factors associated in adolescents in Lima. Design: Analytic transversal study. Material and Methods: A sample of 430 adolescents from one Lima high school was studied. The questionnaire used was the Personal Experience Screening Questionnaire (PESQ). Results: Three hundred and ninety-one valid questionnaires were received. The prevalence of either alcohol or drug use was $43 \%$; the alcohol use $42,2 \%$, marijuana $8,7 \%$, and cocaine $3,1 \%$. The problem of alcohol or drug use was $10,7 \%$. The psychosocial factors associated were psychological distress (OR 4,58 CI 95\% 1,60-13,05), severe thinking problem (OR 2,63 CI 95\% 1,15-6,01), and physical abuse (OR 2,31 CI 95\% 1,19-4,49). Conclusions: The psychosocial factor most associated with drug use was severe psychological distress. Furthermore, an association between both thinking problem and physical abuse with drug use was found. Sexual harassment was found to be associated only in second year high school adolescents.
\end{abstract}

Key words: Substance dependence; street drugs; substance abuse; social problems; alcoholism.

\section{INTRODUCCIÓN}

La adolescencia abarca el periodo entre los 10-19 años de edad (). En Lima Metropolitana, los adolescentes constituyen $21,6 \%$ de la población total, lo que tiende al incremento, sobre todo en las áreas urbanas $\left({ }^{2}\right)$. Durante la adolescencia, se consolida la personalidad y es la etapa en que aparecen las conductas de riesgo. Se entiende por conducta de riesgo aquella que al ser ejecutada con intencionalidad consciente o no, tiene la probabilidad de producir un daño, enfermedad o lesión a 
uno mismo o a los demás. Una de estas conductas de riesgo es el consumo de alcohol y drogas $\left({ }^{3}\right)$. Dentro de los factores que favorecen el consumo de drogas en la adolescencia tenemos aspectos culturales, interpersonales, psicológicos y biológicos, incluyendo la disponibilidad de sustancias, privaciones económicas extremas, comportamiento problemático, bajo rendimiento escolar, inicio precoz de consumo, entre otros $\left({ }^{4}\right)$.

El consumo de alcohol y drogas es una conducta cuya prevalencia presenta actualmente una curva ascendente a nivel mundial. La dependencia de sustancias incluye dos conceptos: dependencia psicológica y dependencia física. La dependencia psicológica enfatiza en las actividades de búsqueda de la sustancia y la evidencia de patrones de uso patológico, y la dependencia física enfatiza en los efectos físicos de los múltiples episodios de uso de la sustancia. Abuso de sustancias, en cambio, está caracterizado por la presencia de al menos un síntoma que indica que el uso de la sustancia interfiere con la vida de la persona $\left({ }^{5}\right)$.

No obstante, Hogan manifiesta en una revisión que no es posible hacer la diferenciación entre uso, abuso y dependencia en los adolescentes, a diferencia de los adultos. Más bien, considera los siguientes síntomas como los más predictivos de un problema de abuso de sustancias en adolescentes: Dejar de hacer otras actividades por usar la droga escogida, continuar usando la droga a pesar de que ocasiona problemas sociales, y conductas de riesgo después del consumo de las sustancias (manejar después de haber ingerido alcohol u otras drogas, etc.) $\left({ }^{6}\right)$. Existen drogas de entrada a drogas más fuertes; proponiéndose los siguientes estadios en el consumo: primero uso de cerveza o vino, luego uso de cigarrillos o licores con alto contenido de alcohol, tercero uso de marihuana y cuarto uso de otras drogas ilícitas $\left({ }^{7}\right)$.
Mientras más joven el adolescente inicia el consumo de alcohol, mayores son las probabilidades de desarrollar un problema de alcoholismo de adulto. Asimismo, el uso de drogas ilícitas muestra un aumento de la prevalencia entre adolescentes en muchos países (). En EU de A, la incidencia de alcohol ha aumentado sostenidamente durante los $90 \mathrm{~s}$ de 3,3 millones de nuevos usuarios en 1990 a 5,6 millones en el 2000, siendo este incremento casi el doble en los menores de 18 años (de 2,2 millones en 1990 a 4,1 millones en el 2000). En el año 2001, 67\% de los nuevos usuarios de marihuana eran menores de 18 años; esta proporción se ha incrementado desde los $60 \mathrm{~s}$, cuando menos de la mitad de los nuevos usuarios era menor de 18 años. El uso de cocaína se ha incrementado sostenidamente en los 90s, alcanzando 1,2 millones en el año 2001. Asimismo, los nuevos usuarios de inhalantes han aumentado de 627000 en 1994 a 1,2 millones en el 2000, siendo éstos predominantemente menores de 18 años $\left(71 \%\right.$ en el 2001) $\left({ }^{8}\right)$. Este consumo tiene como problemas asociados conductas violentas $\left({ }^{9}\right)$ y conductas sexuales de riesgo $\left({ }^{10}\right)$, entre otros.

En la educación secundaria se encuentra la mayor población de adolescentes, lo que da una idea de la prevalencia del consumo de alcohol y drogas y de los factores psicosociales asociados a dicho consumo. Además, en los colegios se puede realizar labor de prevención para este problema.

Los objetivos del presente estudio fueron: 1) determinar la prevalencia del consumo de alcohol y drogas en adolescentes de nivel secundaria de un colegio de Lima, Perú; 2) determinar el porcentaje de los adolescentes que tienen un problema de consumo de alcohol y drogas; 3) determinar la asociación existente entre los factores de riesgo psicosociales y el problema de consumo de alcohol y drogas. 


\section{MATERIAL Y MÉTODOS}

El presente estudio es analítico, de corte transversal. La población evaluada consiste en adolescentes de educación secundaria, de un Colegio Nacional Técnico de Lima Metropolitana. El colegio seleccionado cuenta con aproximadamente 320 adolescentes por año, excepto quinto de secundaria, en donde son aproximadamente 200. La determinación del tamaño de la muestra se calculó mediante la fórmula que utiliza varianza máxima de proporciones, con un nivel de confianza del $95 \%$ y un error máximo permisible de $3 \%$.

Con estos parámetros, se determinó una muestra mínima de 331 adolescentes. El muestreo se realizó por conglomerado, utilizando como unidad de conglomerado las secciones, seleccionando igual número de secciones en cada año de estudios. Se consideró como criterio de inclusión el estar inscrito en el colegio seleccionado, como criterio de exclusión el no haber asistido o el no encontrarse presente al momento de la encuesta. Como criterio de eliminación se consideró el llenado incorrecto de la encuesta.

Se utilizó el Cuestionario Tamiz de Experiencias Personales (PESQ:Personal Experience Screening Questionnaire), el cual cuenta con una sensibilidad del $88 \%$ y una especificidad del $85 \%$ para detectar problema de consumo de alcohol y drogas $\left({ }^{11}\right)$. Esta encuesta consta de 40 preguntas divididas en cinco partes: dos para medir el consumo de alcohol y drogas en los últimos doce meses (severidad del problema y grado de consumo), una para medir los factores psicosociales asociados al consumo y las otras dos para evaluar si los adolescentes pueden estar mintiendo, aumentando el consumo (infrecuencia) o disminuyéndolo (actitud defensiva). Se considera encuesta válida aquella con 39 ó más preguntas respondidas, sin preguntas de infrecuencia positivamente respondidas. Los factores psicosociales analizados han sido divididos en cuatro: distrés psicológico (4 preguntas), pensamiento problemático (2 preguntas), maltrato físico (1 pregunta) y acoso sexual (1 pregunta) $\left({ }^{12}\right)$.

En el presente estudio se consideró las siguientes definiciones:

- Problema de consumo: Consumo de la sustancia más de 10 veces en los últimos 12 meses y/o haber respondido positivamente entre 40 y $50 \%$ de las preguntas de problemas por el consumo de la sustancia, dependiendo de su edad y género.

- Distrés psicológico: Conjunto de conductas y emociones que producen desasosiego en el adolescente. Para el análisis se consideró como distrés psicológico severo el haber respondido positivamente 3 ó 4 de las preguntas de distrés psicológico; como distrés psicológico leve o moderado, el haber respondido positivamente 1 ó 2 de dichas preguntas.

- Pensamiento problemático: Falta de habilidad para la resolución de situaciones problemáticas cotidianas. Se consideró como pensamiento problemático severo el haber respondido positivamente las 2 preguntas de pensamiento problemático, y como pensamiento problemático leve o moderado el haber respondido positivamente 1 de las preguntas.

- Abuso físico: Maltrato físico por algún familiar del adolescente.

- Acoso sexual: Sensación de ser acosado sexualmente.

Para la recolección de datos, se realizó un llenado del PESQ por los adolescentes de educación secundaria de un Colegio Nacional Técnico de Lima Metropolitana con total confidencialidad, de forma anónima, entrega de la encuesta y 
devolución de la misma en sobre cerrado. El tiempo para la resolución de la encuesta fue de 15 minutos. La encuesta fue aplicada por personal de salud, el cual no labora en el colegio evaluado y la recoleccion de los datos se realizó en agosto de 2001.

Se contó con el consentimiento de los adolescentes a participar en el estudio, dándoles la opción de no participar en el mismo, y con la aprobación de la Dirección del Colegio Nacional Técnico.

Para el análisis estadístico, se realizó un análisis de frecuencia para determinar la prevalencia de consumo de alcohol y drogas y la prevalencia del problema de consumo de alcohol y drogas. Se realizó la prueba de $\mathrm{x}^{2}$ en el análisis de la población total y la prueba exacta de Fisher en el análisis por año de estudios, para determinar si existe asociación entre el problema del consumo de alcohol y drogas y la presencia de factores psicosociales, además, se realizó la prueba de OR, con un intervalo de confianza al $95 \%$, para determinar el grado de asociación estadística entre el problema de consumo de alcohol y drogas y los factores psicosociales.

\section{RESULTADOS}

Se encuestó 430 adolescentes, obteniéndose $391(91,4 \%)$ encuestas válidas. El promedio de edad fue 14,4 años (desviación estándar - DE 1,73), siendo $248(63,1 \%)$ de sexo masculino, 123 $(33,1 \%)$ de sexo femenino y $22(3,8 \%)$ no respondieron dicha pregunta. La población por año de estudios fue la siguiente: en primer año $66(16,8 \%)$ adolescentes con una media de edad de 12,8 (DE 1,1), en segundo año $95(24,2 \%)$, con una media de edad de 12,9 (DE 0,9); en tercer año 86 $(21,9 \%)$, con una media de edad de 14,6 (DE 1,1$)$, en cuarto año $84(21,4 \%)$, con una media de edad de 15,4 (DE 0,9$)$ y en quinto año $60(15,3 \%)$, con una media de edad de 16,8 (DE 0,8).

En la Tabla 1 se puede observar el número de estudiantes que ha usado alcohol o drogas en los últimos doce meses $(43 \%)$.

Se halló problema de consumo de alcohol o drogas en 42 adolescentes de la población total $(10,7 \%)$, en primer año 1 ( $1,5 \%$ de la población de dicho año), en segundo año $7(7,4 \%)$, en tercer año 13 $(15,1 \%)$, en cuarto año $11(13,1 \%)$ y en quinto año $10(16,7 \%)$. En las Tablas 2, 3, 4 y 5 , se observa la correlación entre los factores psicosociales y el problema de consumo de alcohol o drogas.

Se halló $150(38,4 \%)$ adolescentes con

Tabla 1. Prevalencia de consumo de alcohol o drogas en los últimos doce meses.

\begin{tabular}{|c|c|c|c|c|c|c|c|c|c|c|c|c|}
\hline & \multicolumn{2}{|c|}{ Primero } & \multicolumn{2}{|c|}{ Segundo } & \multicolumn{2}{|c|}{ Tercero } & \multicolumn{2}{|c|}{ Cuarto } & \multicolumn{2}{|c|}{ Quinto } & \multicolumn{2}{|c|}{ Total } \\
\hline & $\mathrm{n}$ & $(\%)$ & $\mathrm{n}$ & $(\%)$ & $\mathrm{n}$ & $(\%)$ & $\mathrm{n}$ & $(\%)$ & $\mathrm{n}$ & $(\%)$ & $\mathrm{n}$ & $(\%)$ \\
\hline Cualquiera & 13 & $(19,7)$ & 25 & $(26,3)$ & 37 & $(43,0)$ & 49 & $(58,3)$ & 44 & $(73,3)$ & 168 & $(43,0)$ \\
\hline Alcohol & 13 & $(19,7)$ & 24 & $(25,3)$ & 37 & $(43,0)$ & 48 & $(57,1)$ & 43 & $(71,7)$ & 165 & $(42,2)$ \\
\hline Marihuana & 0 & $(0,0)$ & 5 & $(5,3)$ & 8 & $(9,3)$ & 9 & $(10,7)$ & 12 & $(20,0)$ & 34 & $(8,7)$ \\
\hline Cocaína & 0 & $(0,0)$ & 2 & $(2,1)$ & 4 & $(4,7)$ & 1 & $(1,2)$ & 5 & $(8,3)$ & 12 & $(3,1)$ \\
\hline Inhalantes & 0 & $(0,0)$ & 3 & $(3,2)$ & 2 & $(2,3)$ & 2 & $(2,4)$ & 2 & $(3,3)$ & 9 & $(2,3)$ \\
\hline Heroína & 0 & $(0,0)$ & 3 & $(3,2)$ & 1 & $(1,2)$ & 0 & $(0,0)$ & 0 & $(0,0)$ & 4 & $(1,0)$ \\
\hline Anfetaminas & 0 & $(0,0)$ & 2 & $(2,1)$ & 1 & $(1,2)$ & 0 & $(0,0)$ & 0 & $(0,0)$ & 3 & $(0,8)$ \\
\hline Psicodélicos & 0 & $(0,0)$ & 2 & $(2,1)$ & 0 & $(0,0)$ & 0 & $(0,0)$ & 0 & $(0,0)$ & 2 & $(0,5)$ \\
\hline Barbitúricos & 0 & $(0,0)$ & 2 & $(2,1)$ & 0 & $(0,0)$ & 0 & $(0,0)$ & 0 & $(0,0)$ & 2 & $(0,5)$ \\
\hline Tranquilizantes & 0 & $(0,0)$ & 2 & $(2,1)$ & 0 & $(0,0)$ & 0 & $(0,0)$ & 0 & $(0,0)$ & 2 & $(0,5)$ \\
\hline Otros* & 0 & $(0,0)$ & 2 & $(2,1)$ & 0 & $(0,0)$ & 0 & $(0,0)$ & 1 & $(1,7)$ & 3 & $(0,8)$ \\
\hline
\end{tabular}

\footnotetext{
* Otros: Metadona, demerol, opio, morfina, codeína.
} 
Tabla 2. Distrés psicológico y consumo de alcohol y drogas de acuerdo a año de estudios*

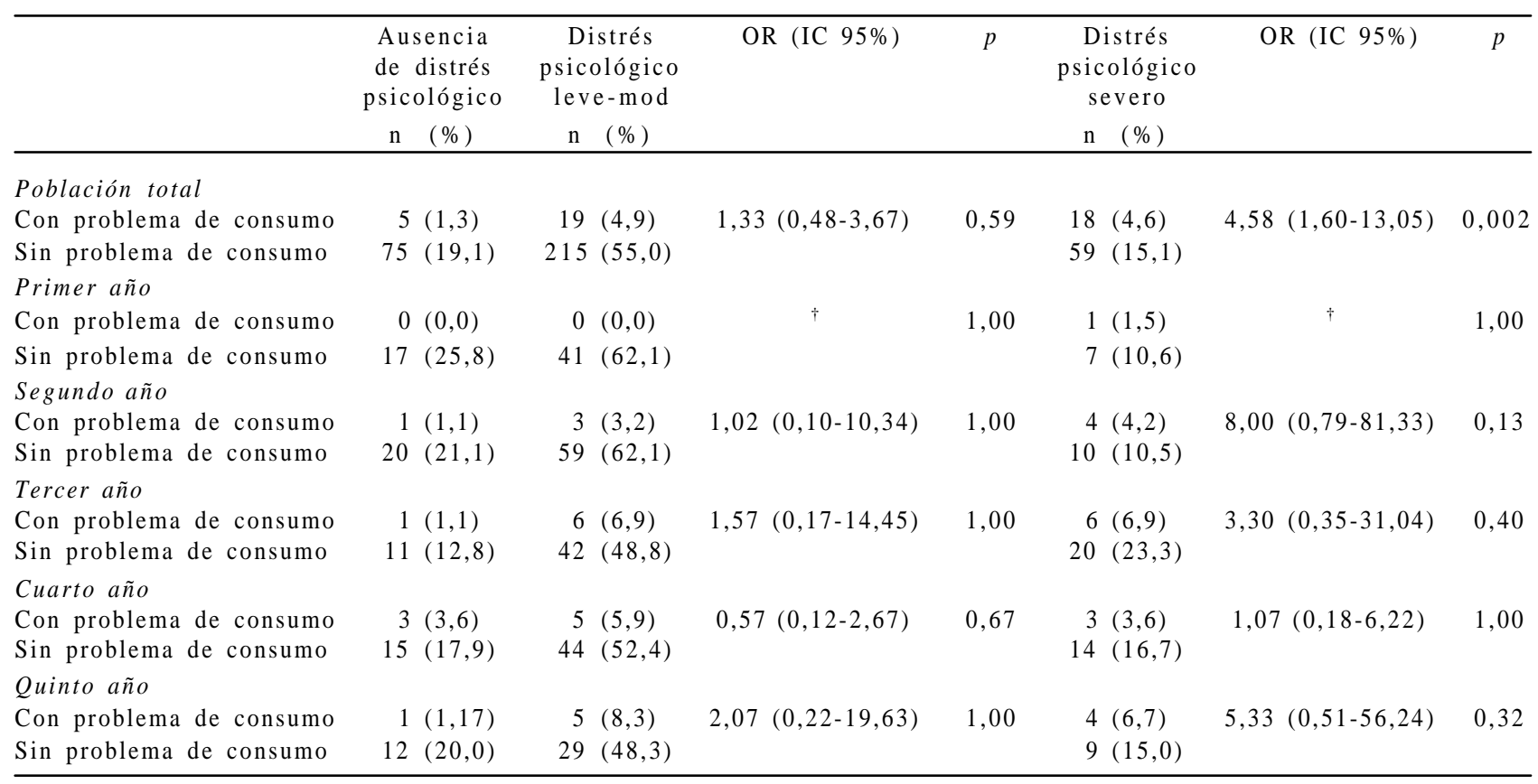

* Para el análisis del distrés psicológico, se asignó a la ausencia de distrés psicológico un OR de 1 y se comparó al grupo de distrés leve o moderado y al grupo de distrés severo contra el de ausencia de distrés.

† En este grupo no se pudo realizar la prueba de odds ratio, dada la distribución de la población.

actitud defensiva hacia la encuesta, en primer año $21(31,8 \%$ de los encuestados de dicho año), en segundo año $42(44,2 \%)$, en tercer año $26(30,2 \%)$, en cuarto año 34 $(40,5 \%)$ y en quinto año $27(45,0 \%)$. No hubo encuestas con preguntas de infrecuencia positivamente respondidas.

\section{DISCUSIÓN}

El consumo de alcohol y drogas se inicia principalmente en la adolescencia $\left({ }^{13}\right)$, grupo etáreo que generalmente cursa educación secundaria.

En el presente estudio se aplicó el PESQ a adolescentes de educación secundaria de un Colegio Nacional Técnico. De acuerdo a los resultados obtenidos, se aprecia que la prevalencia de uso de alcohol y drogas presenta un patrón ascendente a lo largo de los 5 años de estudios, lo cual concuerda con diversos estudios. La mayor prevalencia de consumo es de alcohol
(42,2\% del total), seguido de marihuana $(8,7 \%)$, cocaína $(3,1 \%)$ e inhalantes $(2,3 \%)$, semejante al consumo de alcohol encontrado por Ramírez, el cual evaluó estudiantes de educación secundaria del Callao, Perú $\left({ }^{14}\right)$ y menor que el encontrado por CEDRO, el cual fue $62,3 \%$ en estudiantes de $2^{\circ}, 3^{\circ}$ y $4^{\circ}$ de educación secundaria en Lima $\left({ }^{15}\right)$.

Acerca del consumo de drogas ilegales, la prevalencia encontrada es mayor que la encontrada por CEDRO en 1997, la cual fue de $0,6 \%$ de consumo de marihuana en el último año y $0,4 \%$ de cocaína en adolescentes de 12 a 18 años $\left({ }^{16}\right)$. Sin embargo, CEDRO en una investigación posterior encuentra una prevalencia de consumo de drogas ilegales de $3,6 \%$ en adolescentes de 15 a 17 años $\left({ }^{17}\right)$.

En la Encuesta Nacional de Adicciones (México, 1998), en adolescentes de 12 a 17 años en los últimos 12 meses, se encontró una prevalencia de consumo de 
marihuana en hombres de $1,6 \%$ y en mujeres de $0,3 \%$; de cocaína en hombres de $0,4 \%$ y mujeres de $0,1 \%$; y de inhalantes, en hombres $0,6 \%$ y en mujeres de $0,2 \%\left({ }^{18}\right)$. Rojas-Guiot halló en Pachuca, México, en estudiantes de secundaria y preparatoria de 10 a 22 años, una prevalencia de vida de consumo de alcohol de $47,9 \%$, de inhalantes $2,7 \%$, de marihuana $1,7 \%$ y de cocaína $1,2 \%$, siendo estos resultados menores que los hallados en nuestro estudio, con excepción del consumo de alcohol en Pachuca, probablemente debido al grupo etáreo muestreado $\left({ }^{19}\right)$.

En Brasil, Guimarães encontró una prevalencia de vida de consumo de alcohol de $68,9 \%$, inhalantes $10,0 \%$, marihuana $6,6 \%$ y cocaína $1,6 \%$, en adolescentes escolares de Assis, São Paulo $\left({ }^{20}\right)$. Baus halló una prevalencia de vida de consumo de alcohol de $86,6 \%$, marihuana $19,9 \%$, inhalantes $18,2 \%$ y cocaína $2,9 \%$ en adolescentes escolares de Florianópolis ${ }^{(21)}$. Tavares encontró una prevalencia de consumo de alcohol de 79,6\%, marihuana $8,9 \%$, inhalantes $6,6 \%$ y cocaína $2,4 \%$ en adolescentes de 10 a 19 años, en los últimos 12 meses, de Río Grande do Sul ${ }^{22}$ ) y De Micheli halló una prevalencia de consumo en el último mes de alcohol de $48 \%$, marihuana $14 \%$, inhalantes $5 \%$ y cocaína $3 \%$, en adolescentes escolares de Barueri, São Paulo $\left({ }^{23}\right)$. Estos estudios muestran mayor consumo de alcohol e inhalantes a comparación de los hallados en nuestro estudio. Acerca de cocaína, su consumo es menor y el consumo de marihuana es variable, en promedio similar al hallado en nuestro estudio.

Beyers comunica una prevalencia de consumo, en el último mes, de alcohol en adolescentes de 12 a 14 años de $11,3 \%$ en Victoria (Australia) y 7,1\% en Maine y Oregon (EU de A); y en adolescentes de

Tabla 3. Pensamiento problemático y consumo de alcohol y drogas de acuerdo a año de estudios*

\begin{tabular}{|c|c|c|c|c|c|c|c|c|c|c|}
\hline & $\begin{array}{c}\text { Ause } \\
\text { pens } \\
\text { probl } \\
\mathrm{n} \\
\end{array}$ & $\begin{array}{c}\text { ncia de } \\
\text { amiento } \\
\text { lemático } \\
(\%)\end{array}$ & $\begin{array}{c}\text { Pens } \\
\text { probl } \\
\text { lev } \\
n \\
\end{array}$ & $\begin{array}{l}\text { amiento } \\
\text { lemático } \\
\text { e-mod } \\
(\%)\end{array}$ & OR (IC 95\%) & $p$ & $\begin{array}{c}\text { Pens } \\
\text { probl } \\
\text { se } \\
n \\
\end{array}$ & $\begin{array}{l}\text { amiento } \\
\text { lemático } \\
\text { evero } \\
(\%)\end{array}$ & OR (IC 95\%) & $p$ \\
\hline \multicolumn{11}{|l|}{ Población total } \\
\hline $\begin{array}{l}\text { Con problema de consumo } \\
\text { Sin problema de consumo }\end{array}$ & 11 & $(2,8)$ & $\begin{array}{r}15 \\
175\end{array}$ & $(3,8)$ & $0,87(0,39-1,97)$ & 0,74 & 16 & $(4,1)$ & $2,63(1,15-6,01)$ & 0,02 \\
\hline Primer año & & $(20,0)$ & & (1) & & & & ) & & \\
\hline Con problema de consumo & 0 & $(0,0)$ & 0 & $(0,0)$ & $\dagger$ & 1,00 & 1 & $(1,5)$ & $\dagger$ & 0,46 \\
\hline $\begin{array}{l}\text { Sin problema de consumo } \\
\text { Segundo año }\end{array}$ & 15 & $(22,7)$ & 38 & $(57,6)$ & & & 12 & 18,2 & & \\
\hline Con problema de consumo & & $(0,0)$ & 1 & $(1,1)$ & $\dagger$ & 1,00 & 6 & $(6,3)$ & $\dagger$ & 0,004 \\
\hline Sin problema de consumo & 23 & $(24,2)$ & 53 & $(55,8)$ & & & 12 & $(12,6)$ & & \\
\hline $\begin{array}{l}\text { Tercer año } \\
\text { Con problema de consumo } \\
\text { Sin problema de consumo }\end{array}$ & $\begin{array}{r}4 \\
26\end{array}$ & $\begin{array}{l}(4,7) \\
(30,2)\end{array}$ & $\begin{array}{r}7 \\
34\end{array}$ & $\begin{array}{l}(8,1) \\
(39,5)\end{array}$ & $1,34(0,35-5,06)$ & 0,75 & $\begin{array}{r}2 \\
13\end{array}$ & $\begin{array}{l}(2,3) \\
(15,1)\end{array}$ & $1,00(0,16-6,19)$ & 1,00 \\
\hline $\begin{array}{l}\text { Cuarto año } \\
\text { Con problema de consumo } \\
\text { Sin problema de consumo }\end{array}$ & $\begin{array}{r}4 \\
28\end{array}$ & $\begin{array}{l}(4,8) \\
(33,3)\end{array}$ & $\begin{array}{r}3 \\
32\end{array}$ & $\begin{array}{l}(3,6) \\
(38,1)\end{array}$ & $0,66(0,14-3,19)$ & 0,70 & $\begin{array}{r}4 \\
13\end{array}$ & $\begin{array}{l}(4,8) \\
(15,5)\end{array}$ & $2,15(0,46-9,99)$ & 0,42 \\
\hline $\begin{array}{l}\text { Quinto año } \\
\text { Con problema de consumo } \\
\text { Sin problema de consumo }\end{array}$ & $\begin{array}{r}3 \\
20\end{array}$ & $\begin{array}{l}(5,0) \\
(33,3)\end{array}$ & $\begin{array}{r}4 \\
18\end{array}$ & $\begin{array}{l}(6,7) \\
(30,0)\end{array}$ & $1,48(0,29-7,54)$ & 0,70 & $\begin{array}{r}3 \\
12\end{array}$ & $\begin{array}{l}(5,0) \\
(20,0)\end{array}$ & $1,67(0,29-9,62)$ & 0,66 \\
\hline
\end{tabular}

* Para el análisis del pensamiento problemático se asignó a la ausencia de pensamiento problemático un OR de 1 y se comparó al grupo de pensamiento problemático leve o moderado y al grupo de pensamiento problemático severo contra el de ausencia de pensamiento problemático.

† En este grupo no se pudo realizar la prueba de odds ratio, dada la distribución de la población. 
Tabla 4. Abuso físico y consumo de alcohol y drogas de acuerdo a año de estudios.

\begin{tabular}{|c|c|c|c|c|c|c|}
\hline & $\begin{array}{c}\text { Ausencia } \\
\mathrm{n}\end{array}$ & $\begin{array}{l}\text { abuso físico } \\
(\%)\end{array}$ & $\begin{array}{c}\text { Presencia } \\
\mathrm{n}\end{array}$ & $\begin{array}{l}\text { abuso físico } \\
(\%)\end{array}$ & OR (IC 95\%) & $p$ \\
\hline \multicolumn{7}{|l|}{ Población total } \\
\hline Con problema de consumo & 23 & $(5,9)$ & 18 & $(4,6)$ & \multirow[t]{2}{*}{$2,39(1,19-4,49)$} & \multirow[t]{2}{*}{0,02} \\
\hline Sin problema de consumo & 257 & $(65,7)$ & 95 & $(22,3)$ & & \\
\hline \multicolumn{7}{|l|}{ Primer año } \\
\hline Con problema de consumo & 0 & $(0,0)$ & 1 & $(1,5)$ & \multirow[t]{2}{*}{$*$} & \multirow[t]{2}{*}{0,31} \\
\hline Sin problema de consumo & 45 & $(68,2)$ & 19 & $(28,8)$ & & \\
\hline \multicolumn{7}{|l|}{ Segundo año } \\
\hline Con problema de consumo & 2 & $(2,1)$ & 5 & $(5,3)$ & \multirow{2}{*}{$5,00(0,91-27,35)$} & \multirow{2}{*}{0,09} \\
\hline Sin problema de consumo & 58 & $(61,1)$ & 29 & $(30,5)$ & & \\
\hline \multicolumn{7}{|l|}{ Tercer año } \\
\hline Con problema de consumo & 6 & $(6,9)$ & 7 & $(8,1)$ & \multirow[t]{2}{*}{$4,08(1,20-13-88)$} & \multirow[t]{2}{*}{0,04} \\
\hline Sin problema de consumo & 56 & $(65,1)$ & 16 & $(18,6)$ & & \\
\hline \multicolumn{7}{|l|}{ Cuarto año } \\
\hline Con problema de consumo & 8 & $(9,5)$ & 3 & $(3,6)$ & \multirow[t]{2}{*}{$1,24(0,29-5,18)$} & \multirow[t]{2}{*}{0,72} \\
\hline Sin problema de consumo & 56 & $(66,7)$ & 17 & $(20,3)$ & & \\
\hline \multicolumn{7}{|l|}{ Quinto año } \\
\hline Con problema de consumo & 7 & $(11,7)$ & 2 & $(3,3)$ & \multirow{2}{*}{$2,00(0,33-11,97)$} & \multirow[t]{2}{*}{0,60} \\
\hline Sin problema de consumo & 42 & $(70,0)$ & 6 & $(10,0)$ & & \\
\hline
\end{tabular}

* En este grupo no se pudo realizar la prueba de odds ratio, dada la distribución de la población.

15 a 17 años de $32,4 \%$ en Australia y $20,5 \%$ en EU de A. Asimismo, se halló una prevalencia de consumo de marihuana en adolescentes de 12 a 14 años de $6,7 \%$ en EU de A y 3,8\% en Australia; y en adolescentes de 15 a 17 años de $23,1 \%$ en EU de A y $16,1 \%$ en Australia $\left({ }^{24}\right)$.
El problema de consumo en nuestro estudio presenta un patrón ascendente en los tres primeros años, siendo de $10,7 \%$ a nivel global, a partir del $3^{\circ}$ tiende a estabilizarse. Soldera, en Brasil, encuentra un uso pesado de drogas, definido como el uso de sustancias en 20 días o más del

Tabla 5. Acoso sexual y consumo de alcohol y drogas de acuerdo a año de estudios.

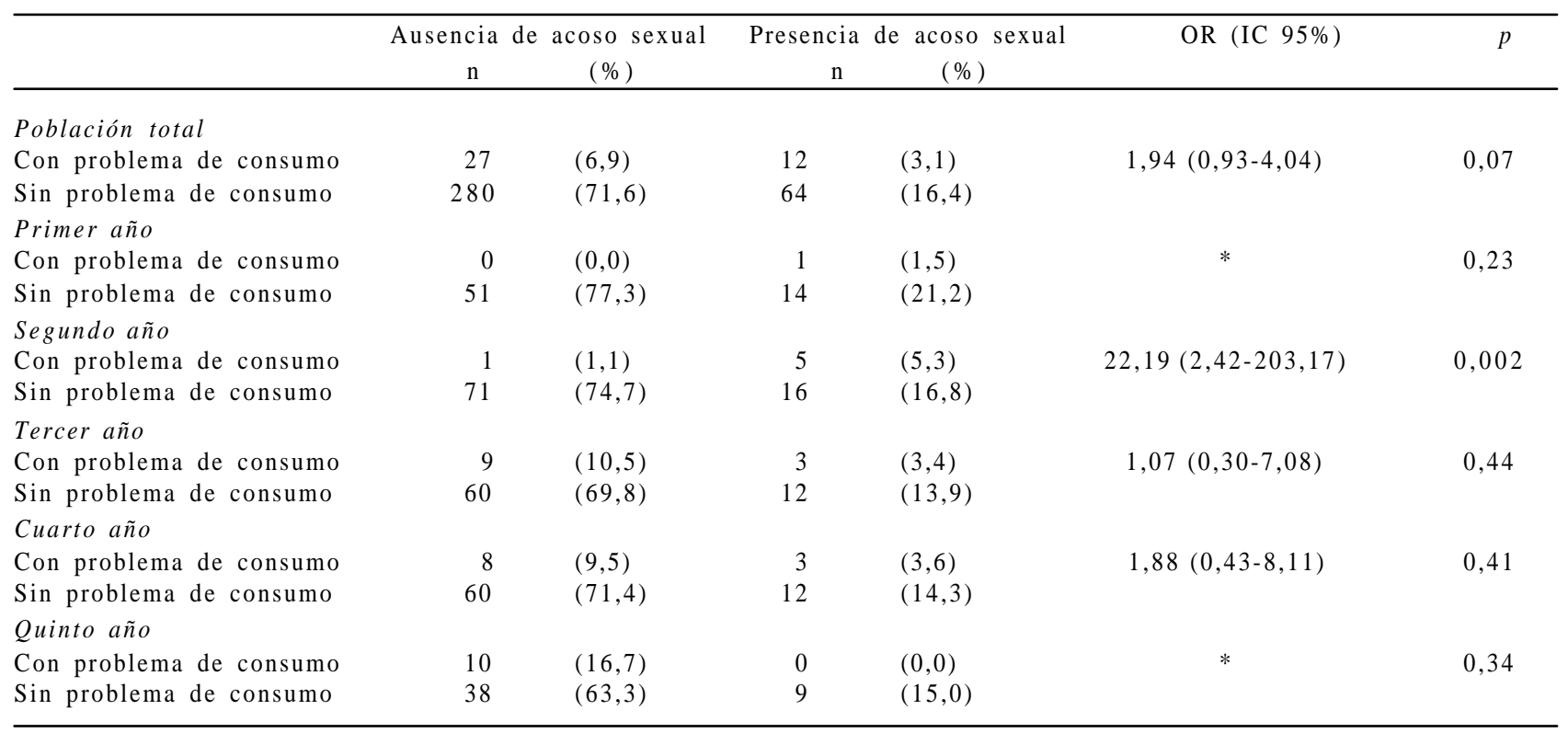

* En este grupo no se pudo realizar la prueba de odds ratio, dada la distribución de la población. 
último mes, para alcohol de $11,9 \%$, marihuana $4,4 \%$, inhalantes $1,8 \%$ y cocaína $1,4 \%\left({ }^{25}\right)$, mayor que el problema de consumo hallado en nuestro estudio.

Con respecto al distrés psicológico, la prevalencia de este factor entre los encuestados se dividió en tres grupos: un grupo que no presenta distrés psicológico, un grupo con distrés psicológico grado leve-moderado, que tiene una prevalencia mayor en general y cuya magnitud no tiende a variar mucho en cada año, y un grupo con distrés psicológico severo, que tiene una prevalencia menor en los resultados, pero cuya presentación es más heterogénea en cada año, en mayor porcentaje en $3^{\circ}$, en el que es superior comparado con los dos primeros años. Este mayor porcentaje en la prevalencia del distrés psicológico severo coincide con el aumento en la prevalencia del problema de consumo de alcohol y drogas. En el grupo con distrés psicológico levemoderado no hay una asociación estadísticamente significativa con el problema de consumo de alcohol. En el grupo con distrés psicológico severo, sí se observa una asociación estadísticamente significativa. Este distrés psicológico reflejaría sentimientos de frustración y problemas en los adolescentes que los haría más propensos a adoptar conductas de riesgo, como el consumo de alcohol y drogas.

Acerca del pensamiento problemático, se subdividió en tres grupos: uno que no presenta pensamiento problemático, otro con grado leve-moderado y otro con grado severo. El segundo grupo presenta mayor prevalencia en todos los años, pero va descendiendo de $1^{\circ}$ a $5^{\circ}$. En el grupo de grado severo con menor prevalencia, a comparación del anterior, se encuentran porcentajes menores en los tres primeros años y mayores en los dos últimos. El grupo con pensamiento problemático en grado severo presentó asociación estadísticamente significativa con el problema de consumo de alcohol y drogas, aunque en menor grado que el distrés psicológico.

El abuso físico es un factor que refleja una mala relación del adolescente con su entorno familiar y problemas de comunicación con sus padres, y esta mala relación con los padres se halla asociada al consumo de alcohol y drogas $\left({ }^{19}\right)$. Este factor presenta una prevalencia que va disminuyendo de $1^{\circ}$ al $5^{\circ}$, lo que no necesariamente significa una mejor relación con el entorno familiar. En nuestro estudio, tiene una asociación estadísticamente significativa con el problema de consumo de alcohol y drogas. Este factor tiene un mayor grado de asociación en $3^{\circ}$, lo cual podría explicar parcialmente el incremento del problema de consumo en dicho año.

El acoso sexual es un factor que podría llevar a situaciones de temor y frustración; es importante ver que el porcentaje de prevalencia de este factor también va disminuyendo de $1^{\circ}$ a $5^{\circ}$ en forma sostenida. En el resultado general, este factor no tiene asociación estadísticamente significativa: sin embargo, cabe resaltar que en $2^{\circ}$ año existe una fuerte asociación estadística (OR 22,2 ), lo que nos podría indicar que este factor sería importante para la aparición del problema de consumo de alcohol y drogas. Chandy encontró una asociación entre el abuso sexual y el consumo de alcohol en ambos sexos en poblaciones adolescentes y un incremento en el consumo de marihuana en los varones $\left({ }^{26}\right)$.

También se evaluó la actitud defensiva de los adolescentes ante la encuesta; un alto porcentaje presentó actitud defensiva $(38,5 \%)$, indicando que existiría un grupo de estudiantes que minimizan su consumo.

La conclusiones de nuestro estudio fueron:

1. La prevalencia de uso de cualquier sustancia fue del 43,0\%, siendo las más frecuentes el alcohol con $42,2 \%$ y la marihuana con $8,7 \%$. 
2. La prevalencia de problema de consumo de alcohol y drogas fue $10,7 \%$, siendo este problema mayor en los últimos años.

3. El factor psicosocial más asociado al problema de consumo de drogas fue el distrés psicológico severo. Asimismo, se encontró asociación entre el pensamiento problemático severo y el abuso físico con dicho problema. El acoso sexual sólo se encontró asociado en los adolescentes de $2^{\circ}$ de secundaria

Finalmente, cabe mencionar que el problema de consumo de alcohol y drogas constituye una situación determinada por múltiples factores adicionales no tomados en cuenta de forma directa en este estudio. Se recomienda el desarrollo de estudios más profundos y detallados para ahondar en el problema de consumo de alcohol y drogas en adolescentes.

\section{AGRADECIMIENTOS}

Al Dr. Ken C. Winters, PhD, por su colaboración y apoyo en la realización del presente estudio.

\section{REFERENCIAS BIBLIOGRÁFICAS}

1. The Department of Child and Adolescent Health and Development. [homepage on the Internet]. Geneva: World Health Organization; c2000-2004 [cited 2004 Jul 25]. Overview of CAH; [about 2 screens]. Available from: http:/ /www.who.int/child-adolescent-health/over.htm

2. IX Censo Nacional de Población y IV de Vivienda 1993 [database on the Internet]. Lima: Instituto Nacional de Estadística e Informática. c2002 - [cited 2004 Jul 25]. Available from: http://www.inei.gob.pe/biblioinei.asp

3. Perales A, Sogi C, Sánchez E. Conductas de Riesgo en Adolescentes de Lima. Monografías de Investigación No 07. 1era ed. Lima: Instituto Nacional de Salud Mental Honorio Delgado-Hideyo Noguchi; 1999.

4. Marques AC, Cruz M. O adolescente e o uso de drogas. Rev Bras Psiquiatr. 2000;22 Supl II:32-6.

5. Sadock BJ. Substance related disorders. En: Sadock BJ, Sadock VA, editors. Kaplan and Sadock's Synopsis of Psychiatry: Behavioral Sciences Clinical Psychiatry. Lippincott Williams \& Wilkins; 2002. p. 380-470.

6. Hogan M. Diagnosis and treatment of teen drug use. Med Clin of Nort Am. 2000;84:927-66.
7. Kaminer Y. Addictive disorders in adolescent. Psychiat Clin Nort Am. 1999;22:275-89.

8. Results from the 2002 National Survey on Drug Use and Health: National Findings [database on the Internet]. Washington: Substance Abuse and Mental Health Services Administration. c2000 - [cited 2004 Jul 25]. Available from: http://www.oas.samhsa.gov/nhsda/2k2nsduh/Results/ 2k2Results.htm\#toc

9. Grann M, Fazel S. Substance misuse and violent crime: Swedish population study. BMJ. 2004;328:1233-4.

10. Rashad I, Kaestner R. Teenage sex, drugs and alcohol use: problems identifying the cause of risky behaviors. J Health Economics. 2004;23:493-503.

11. Winters KC. Development of an adolescent alcohol and others drug abuse screening scale: personal experience screening questionnaire. Addict Behav. 1992;17:479-90.

12. Henly GA, Winters KC. Development of psychosocial scales for the assessment of adolescent involved with alcohol and drugs. Int J Addict. 1988;24:973-1001.

13. Estadísticas sobre producción, tráfico y consumo de drogas 1997-1998 [database on the Internet]. Lima: Instituto Nacional de Estadística e Informática. c2002 - [cited 2004 Jul 25]. Available from: http://www.inei.gob.pe/biblioinei.asp

14. Ramírez R. Estudio Epidemiológico-Toxicológico del uso y abuso de sustancias psicoactivas en adolescentes del Callao. [Trabajo de Aptitud Profesional para optar por el título de Químico-Farmacéutico]. Universidad Nacional Mayor de San Marcos. Lima Perú. 1995.

15. Zavaleta A. El problema de las drogas en el Perú 2003. Lima: CEDRO; 2003.

16. CEDRO-Área de Investigaciones. Epidemiología de drogas en la población urbana peruana 1997-Encuesta Nacional de Hogares. Monografía de Investigación $N^{\circ}$ 17. Lima (Perú): CEDRO; 1997.

17. Zavaleta A, Romero E, Castro de la Mata R. Variables asociadas a la prevalencia de vida de drogas en jóvenes de Lima-Perú. En: Zavaleta A, editor. Factores de riesgo y protección en el consumo de drogas en la juventud. Lima: CEDRO; 2001. p. 29-50.

18. Medina-Mora ME, Cravioto P, Villatoro J, Fleiz C, GalvánCastillo F, Tapia-Conyer R. Consumo de drogas entre adolescentes: Resultados de la Encuesta Nacional de Adicciones 1998. Salud Publica Mex. 2003;45 Supl 1:S1625.

19. Rojas-Guiot E, Fleiz-Bautista C, Medina-Mora ME, Moron M, Domenech-Rodríguez M. Consumo de alcohol y drogas en estudiantes de Pachuca, Hidalgo. Salud Publica Mex. 1999;41:297-308.

20. Guimarães JL, Godinho PH, Cruz R, Kappann JI, Tosta LA. Consumo de drogas psicoativas por adolescentes escolares de Assis, SP. Rev Saúde Pública. 2004;38(1):130-2.

21. Baus J, Kupek E, Pires M. Prevalência e factores de risco relacionados ao uso de drogas entre escolares. Rev Saúde Pública. 2002;36(1):40-6.

22. Tavares BF, Béria JU, Silva de Lima M. Prevalência do uso de drogas e desempenho escolar entre adolescentes. Rev Saúde Pública. 2001;35(2):150-8.

23. De Micheli D, Formigoni ML. Drug use by Brazilian students: associations with family, psychosocial, health, 
demographic and behavioral characteristics. Addiction. 2004;99:570-8.

24. Beyers JM, Toumbourou JW, Catalano RF, Arthur MW et al. A Cross-national comparison of risk and protective factors for adolescent substance use: The United States and Australia. J Adolesc Health. 2004;35:3-16.

25. Soldera M, Dalgalarrondo P, Corrêa Filho H, Silva C. Uso de drogas psicotrópicas por estudantes: Prevalência e fatores sociais associados. Rev Saúde Pública. 2004;38(2):277-83.

26. Chandy JM, Blum R, Resnick, MD. Gender-specific outcomes for sexually abused adolescents. Child Abuse Negl. 1996;20:1219-31.

Correspondencia:

Dr. Manuel Francisco Ugarte Gil

Calle Loma Verde 383, Santiago de Surco

Lima 33, Perú

Correo-e: manuel.ugarte@edhucasalud.org 